\title{
861.
}

\section{NOTE ON A FORMULA RELATING TO THE ZERO-VALUE OF A THETA-FUNCTION.}

[From Crelle's Journal der Mathem., t. c. (1887), pp. 87, 88.]

I HAD some difficulty in verifying for the case of a single theta-function, a formula given in Herr Thomae's paper "Beitrag zur Theorie der 9-Functionen," Crelle's Journal, vol. LXXI. (1870), pp. 201-222. The formula in question (see p. 216) is given as follows :

$$
9(0,0, \ldots 0)=\sqrt{\frac{\left|A_{\lambda}^{\left(\lambda^{\prime}\right)}\right|}{(2 \pi i)^{p}}} \sqrt[4]{\text { Discr. }(0,0, \ldots 0) \text { Discr. }(0,0, \ldots 0)}
$$

but the denominator factor should I think be $(\pi i)^{p}$ instead of $(2 \pi i)^{p}$. Making this alteration, then in the case of a single theta-function, $p=1$, and the function belongs to the radical

where

$$
\sqrt{x-k_{1} \cdot x-k_{2} \cdot x-k_{3} \cdot x-k_{4}},
$$

$$
\left(k_{1}, k_{2}, k_{3}, k_{4}\right)=\left(-\frac{1}{k},-1,+1,+\frac{1}{k}\right) \text {. }
$$

The determinant $\left|A_{\lambda}{ }^{\left(\lambda^{\prime}\right)}\right|$ is a single term $=A$, and the formula becomes

$$
\vartheta(0)=\sqrt{\frac{A}{\pi i}} \cdot \sqrt[4]{\left(k_{3}-k_{1}\right)\left(k_{4}-k_{2}\right)},
$$

where $k_{3}-k_{1}, k_{4}-k_{2}$ are each $=1+\frac{1}{k}$, and we have therefore

also $A$ denotes the integral

$$
\vartheta(0)=\sqrt{\frac{A}{\pi i}\left(1+\frac{1}{k}\right)}
$$

$$
\begin{aligned}
\int_{k_{1}}^{k_{2}} \frac{d x}{\sqrt{x-k_{1} \cdot x-k_{2} \cdot x-k_{3} \cdot x-k_{4}}}, & =\int_{-\frac{1}{k}}^{-1} \frac{k d x}{\sqrt{1-x^{2} \cdot 1-k^{2} x^{2}}} \\
& =\int_{1}^{\frac{1}{c}} \frac{k d x}{\sqrt{1-x^{2} \cdot 1-k^{2} x^{2}}}=i k K^{\prime}
\end{aligned}
$$


and the formula thus is

$$
9(0)=\sqrt{\frac{K^{\prime}(1+k)}{\pi}}
$$

But observe that, in the theta-function as defined by the equation

$$
\vartheta(x)=\Sigma e^{a m^{2}+2 m x},
$$

$a$ is used to denote the value

$$
a=a_{1}^{\prime} B,=\frac{2 \pi}{A} B
$$

where $A$ is the above-mentioned integral, and $B$ is the integral

$$
B=\int_{k_{2}}^{k_{3}} \frac{d x}{\sqrt{x-k_{1} \cdot x-k_{2} \cdot x-k_{3} \cdot x-k_{4}}}, \quad=\int_{-1}^{1} \frac{k d x}{\sqrt{1-x^{2} \cdot 1-k^{2} x^{2}}}=2 k K,
$$

which value must however be taken negatively, viz. we must write $B=-2 k K$, and we then have

viz. writing as usual

$$
a=-\frac{2 \pi K}{K^{\prime}}
$$

$$
q=e^{-\frac{\pi K^{\prime}}{K}}, \quad r=e^{-\frac{\pi K}{K^{\prime}}}
$$

the $e^{a}$ of the theta-function is not $=q$, but it is $=r^{2}$; and the zero-value $9(0)$ is $=1+2 r^{2}+2 r^{8}+2 r^{18}+\ldots$. The equation thus is

$$
1+2 r^{2}+2 r^{8}+2 r^{18}+\ldots=\sqrt{\frac{K^{\prime}(1+k)}{\pi}}
$$

which is right; in fact, writing $k^{\prime}$ in place of $k$, and consequently $K, q$ in place of $K^{\prime}, r$ respectively, the equation becomes

we have

$$
1+2 q^{2}+2 q^{8}+2 q^{18}+\ldots=\sqrt{\frac{K\left(1+k^{\prime}\right)}{\pi}}
$$

$$
1+2 q+2 q^{4}+2 q^{9}+\ldots=\sqrt{\frac{2 K}{\pi}}
$$

and changing $q$ into $q^{2}$, then (Fund. Nova, p. 92) $K$ is changed into $\frac{K\left(1+k^{\prime}\right)}{2}$, and we have the formula in question. As a verification for small values of $q$, observe that we have

$$
\frac{2 K}{\pi}=1+4 q+4 q^{2}, \quad \frac{1+k^{\prime}}{2}=1-4 q+16 q^{2}
$$

and thence

$$
\frac{K\left(1+k^{\prime}\right)}{\pi}=1+4 q^{2} \text { or } \sqrt{\frac{K\left(1+k^{\prime}\right)}{\pi}}=1+2 q^{2}
$$

Cambridge, 12 February, 1886. 